\title{
Maternal quality and safety outcomes for Asians and Pacific Islanders in Hawai'i: an observational study from five years of statewide data
}

Tetine Sentell $^{1 *}$, Ann Chang ${ }^{2}$, Yongjun Cheng ${ }^{3}$ and Jill Miyamura ${ }^{4}$

\begin{abstract}
Background: Empirical evidence regarding maternal quality and safety outcomes across heterogeneous Asian and Pacific Islanders subgroups in the United States is limited, despite the importance of this topic to health disparities research and quality improvement efforts.

Methods: Detailed discharge data from all Hawai'i childbirth hospitalizations ( $n=75,725)$ from 2008 to 2012 were considered. Validated measures of maternal quality and safety were compared in descriptive and multivariable models across seven racial/ethnic groups: Filipino, Native Hawaiian, other Pacific Islander (e.g., Samoan, Tongan, Micronesian), Japanese, Chinese, white, and other race/ethnicity. Multivariable models adjusted for age group, payer, rural vs. urban hospital location, multiple gestation, and high-risk pregnancy.
\end{abstract}

Results: Compared to whites, Japanese, Filipinos, and Other Pacific Islanders had significantly higher overall delivery complication rates while Native Hawaiians had significantly lower rates. Native Hawaiians also had significantly lower rates of obstetric trauma in vaginal delivery with and without instruments compared to whites (Rate Ratio (RR):0.66; 95\% Cl:0.50-0.87 and RR:0.62; 95\% Cl:0.52-0.74, respectively). Japanese and Chinese had significantly higher rates of obstetric trauma for vaginal deliveries without instruments (RR:1.52; 95\% Cl:1.27-1.81 and RR:1.95;95\% Cl:1.53-2.48, respectively) compared to whites, and Chinese also had significantly higher rates of birth trauma in vaginal delivery with instrument (RR 1.42; 95\% Cl:1.06-1.91). Filipinos and Other Pacific Islanders had significantly higher rates of Cesarean deliveries compared to whites (RR:1.15; 95\% Cl:1.11-1.20 and RR:1.16; 95\% Cl:1.10-1.22, respectively). Other Pacific Islanders also had significantly higher rates of vaginal births after Cesarean (VBAC) deliveries compared to whites (RR: 1.28; 95\% Cl:1.08-1.51) and Japanese had significantly lower rates of uncomplicated VBACs (RR:0.77; 95\% Cl:0.63-0.94).

Conclusions: Significant variation was seen for Asian and Pacific Islander subgroups across maternal quality and safety outcomes. Notably, high rates of obstetric trauma were seen among Chinese and Japanese vaginal deliveries. Filipinos and other Pacific Islanders had high rates of Cesarean deliveries. Native Hawaiians had better quality and safety outcomes than whites on several quality and safety measures, including obstetric trauma during vaginal delivery. Other Pacific Islanders had high rates of VBACs, while Japanese had lower rates. This information can help guide clinical practice, research, and quality improvement efforts.

Keywords: Asian, Pacific Islanders, Obstetrics, Health care quality, Cesarean

\footnotetext{
* Correspondence: tsentell@hawaii.edu

'Office of Public Health Studies, University of Hawai'i at Mānoa, 1960

East-West Road, Biomed T102, Honolulu, HI 96822, USA

Full list of author information is available at the end of the article
} 


\section{Background}

Childbirth is the most common reason women are hospitalized in the United States (U.S.) [1]. Childbirth hospitalizations represent a major expense for public and private insurers with a total cost of over \$15 billion a year [1]. Despite their high costs, U.S. maternal and child outcomes are worse than most other developed countries across such critical factors as maternal and infant mortality and low birth weight $[2,3]$, and are notably unequal by race/ethnicity $[4,5]$. For these reasons, pregnancy and childbirth has been identified as a national priority area for health care quality improvement [6]. Health care quality has been defined by the Institute of Medicine (IOM) as "safe, effective, patient-centered, timely, efficient, and equitable" [7]. Safety, the prevention of harm to patients from their interaction with the health care system, has been called "the foundation upon which all other aspects of quality care are built" [8].

Asians and Pacific Islanders are rapidly growing racial/ ethnic groups in the U.S. [9] with particularly large populations in some states, such as California and Hawai'i, and in some communities, including New York City [10]. Birth outcomes are extremely understudied in these populations [11-14]. Many studies that do include Asian and Pacific Islanders often combine heterogeneous subpopulations due to sample size limitations, likely masking important differences [15-17]. The few studies that conduct detailed comparisons of Asian and Pacific Islander subgroups in the U.S. find significant differences in maternal health outcomes across subgroups [13,14]. For instance, Filipinos have particularly high rates of lacerations [18], Native Hawaiians have notably high rates of preterm delivery [14], and macrosomia is higher in Pacific Islander women [12]. However, detailed data about many important health outcomes, including childbirth quality and safety, are limited.

The Agency for Healthcare Research and Quality (AHRQ) has developed tools to measure obstetric care quality and safety based on administrative health data [19]. These reflect potentially preventable adverse events and are widely used local, nationally, and internationally [20]. These metrics have many advantages, including extensive measurement development and refinement, integrated risk-adjustment, comparability across multiple locations, and national benchmarks [19-21].

Specifically, the AHRQ metrics are grouped into "quality" and "safety" categories. While associated, these are designed to capture distinct health care system concerns [21]. Inpatient Quality Indicators are measures of factors such as volume and utilization designed to help hospitals identify potentially problematic areas of use [21]. The four AHRQ Inpatient Quality Indicators measures focused on obstetric conditions are the Cesarean delivery rate, the primary Cesarean delivery rate, the Vaginal Birth
After Cesarean (VBAC) rate, uncomplicated, and the VBAC, all rate. Patient Safety Indicators are designed to "capture adverse events that patients experience as a result of exposure to the health care system" [19], such as complications and adverse events following surgeries and childbirth. The three AHRQ Inpatient Patient Safety Indicators measures focused on obstetric conditions are the obstetric trauma rate from: 1. Cesarean delivery, 2. Vaginal delivery without instrument, and 3. Vaginal delivery with instrument.

Existing studies have not comprehensively considered these measures across Asian American and Pacific Islander subgroups [15]. This information can provide important benchmarks for quality improvement efforts, especially for locations with large populations of heterogeneous Asian and Pacific Islander groups, and can identify areas of priority and urgency [22]. It also provides data to allow for evidence-based clinical discussions of perinatal risk specific to Asian and Pacific Islander subgroups [12].

The study goal was to fill a key research gap by comparing AHRQ maternal quality and safety outcomes across Asian American and Pacific Islander groups in Hawai i, specifically across seven racial/ethnic groups: Filipino, Native Hawaiian, other Pacific Islander (e.g., Samoan, Tongan, Micronesian), Japanese, Chinese, other race/ ethnicity and white (as comparison). Our hypothesis was that we would see poorer maternal quality and safety outcomes for Native Hawaiians, Filipinos, and other Pacific Islanders compared to whites. This was based on a large literature showing higher risk for Native Hawaiians, Filipinos, and other Pacific Islanders compared to whites across multiple domains associated with poor maternityrelated outcomes (e.g., smoking, lower socioeconomic status, higher rates of chronic disease) as well as poorer access to care [23-25].

\section{Methods \\ Data}

Detailed discharge data from all Hawai'i childbirth hospitalizations from 2008 to 2012 were considered using the Hawai'i Health Information Corporation (HHIC) inpatient all-payer data source [26]. These hospitalizations come from the 15 hospitals in Hawai'i reporting at least one delivery during this period. Data elements include patient race/ethnicity, age, gender, insurer, length of stay, and diagnosis (based on International Classification of Diseases $9^{\text {th }}$ revision - Clinical Modification (ICD-9) codes and Medicare Severity-Diagnostic Related Groups (MS-DRGs)). HHIC data links mothers to newborns and includes a unique patient identifier for individuals across all hospitals.

\section{Sample}

We began with the 89,263 vaginal or Cesarean section delivery hospitalizations in Hawai'i between January 
2008-December 2012. Hospitalizations from Tripler, the Department of Defense (DOD) hospital $(n=10,525)$, were then excluded as this hospital does not consistently report racial/ethnic data. Any other hospitalizations missing race/ethnicity data $(\mathrm{n}=3,003)$ and hospitalizations for payer type as miscellaneous $(n=10)$ were also excluded. The final sample was 75,725 hospitalizations from 62,316 women.

\section{Outcome variables}

AHRQ measures

Using published guidelines [19], we considered four AHRQ maternal-specific inpatient quality indicators. These were the Cesarean delivery rate, the primary Cesarean delivery rate, Vaginal Birth After Cesarean (VBAC) rate, uncomplicated, and VBAC, all. We also considered the three AHRQ maternal-specific inpatient safety measures [15]. These were the obstetric trauma rates from: 1. Cesarean delivery, 2. Vaginal delivery without instrument, and 3. Vaginal delivery with instrument. The specific diagnostic codes used to determine these rates are found in Table 1 .

\section{Total maternal complications}

We also considered a measure of positive maternal outcomes. To do this, we followed the methods of Gregory et al. (2009) who suggested that a useful metric, easily calculated and readily understood by health care consumers, could be based on the proportion of women delivering without maternal or newborn childbirth morbidity [27]. Their metric, which they termed the "ideal delivery (ID) rate", measures the proportion of positive birth outcomes from total deliveries. We followed their published guidelines [27] to calculate maternal or newborn childbirth morbidity, identified from the ICD-9 codes indicating 26 possible problematic outcomes, including bladder lacerations, pelvic hematoma, neonatal birth trauma, and neonatal respiratory problems. The rates of ideal deliveries (those that did not include the problematic outcomes) were calculated for each Asian and Pacific Islander subgroup. The diagnostic codes specifically used to create these rates are found in Table 1.

\section{Race/ethnicity}

Outcomes were compared in descriptive and multivariable models across seven racial/ethnic groups: Filipino, Native Hawaiian, other Pacific Islander (e.g., Samoan, Tongan, Micronesian), Japanese, Chinese, white, and other race/ethnicity using HHIC race/ethnicity classifications. The race/ethnicity variable is created from the race/ethnicity categories available across all hospitals [26]. HHIC performs extensive ongoing quality assurance to confirm that racial/ethnic data are uniformly collected across all Hawai'i hospitals. Only one primary race is reported by hospitals from patient self-report at intake, so mixed race is not available. Mixed-race individuals are represented by self-report of their primary race of identification or were included in the "other" racial/ethnic category if patients did not wish to choose one primary $\mathrm{racial} / \mathrm{ethnic}$ identification.

\section{Control variables}

Based on previous research [4,5,11-14], we included the following variables in our multivariable models to control for their impact on health outcomes by race/ ethnicity: age $(<20 ; 20-29 ; 30-30 ; 40+)$, payer (Department of Defense, private, self-pay or public), high-risk (yes or no), and location of the hospital (urban vs. rural). Public insurance included Medicare and Medicaid. High risk was measured using previous research [27] and included measures of factors such as chronic disease and substance abuse. Location of the hospital (urban vs. rural) was included due to the expectations of more limited resources and distinct case mixes in rural hospitals. Multiple gestation was also included as a control variable when relevant. (Following the AHRQ metrics [19], births for multiple gestation are excluded from the measurement of Cesarean delivery rate, primary Cesarean delivery rate, and Vaginal Birth After Cesarean (VBAC) rate, uncomplicated.) We also included newborn birth weight in three categories: <2500 grams (low birth weight), between 2500-4000 grams (normal birth weight), and $>4000$ grams (high birth weight) in control models.

\section{Statistical methods}

We first summarized outcomes and control variables in descriptive statistics by race/ethnicity using Chi-squared tests or Fisher's exact tests (for categorical variables) and analysis of variance (ANOVA) or non-parametric Kruskal-Wallis tests, if the normality assumption was not satisfied (for continuous variables). Multivariable generalized linear models using generalized estimating equations (GEE) with binomial distribution, log link function and autoregressive of first order $(\operatorname{AR}(1))$ as working correlation matrices were then used to estimate rate ratios (RR) by race/ethnicity with $95 \%$ confidence intervals (CI).

Demographic analyses were based on an individual's first visit. Other analyses were at the level of hospitalization. Deliveries with twins or multiples had only one hospitalization, but individuals could have multiple deliveries during the study period for separate pregnancies. Statistical models accounted for these repeated measures using GEE. All multivariable models adjusted for age group, payer, rural vs. urban hospital location, high-risk pregnancy and newborn birth weight.

Multiple gestation was included when relevant as described above. Two-sided p-value $<0.05$ was regarded as statistically significant. All analyses were performed in 


\section{Table 1 MS-DRG and ICD-9-CM codes used to define each maternal health indicator}

\section{Maternal quality indicator}

Cesarean delivery rate

Numerator

MS-DRG: 765, 766

Primary Cesarean delivery rate

MS-DRG: 765,766

MS-DRG: 767, 768, 774, 775

Vaginal Birth After Cesarean, all rate

MS-DRG: 767, 768, 774, 775

Vaginate
rate

Obstetric Trauma Rate - Vaginal Delivery Without Instrument

Obstetric Trauma Rate - Vaginal Delivery With Instrument

Obstetric Trauma Rate - Cesarean delivery

\section{Non-complicated deliveries}

Ideal Delivery Rate '728','729'
MS-DRG: $765,766,767,768,774,775$

MS-DRG: 765, 766, 767, 768, 774, 775

MS-DRG: 767, 768, 774, 775 and diagnosis code '66420', '66421', '66424', '66430', '66431', '66434'

MS-DRG: 767, 768, 774, 775, diagnosis code: '66420', '66421', '66424', '66430', '66431','66434', and procedure codes: '720','721','7221','7229', '7231','7239','724', '7251','7253','726','7271','7279'

MS-DRG: 765, 766 and diagnosis codes: '66420','66421','66424','66430','66431', '66434'

Diagnosis code not in $(664.2,664.3665 .5665 .4$ $664.4,664.8,664.9,665.8,665,665.1,674.1,666.0$ $664.4,664.8,664.9,665.8,665,665.1,674.1,666.0$
$666.1,666.2,666.3,665.7,664.5,670 . x x, 038 . x x$, $666.1,666.2,666.3,665.7,664.5,670 . x x, 038 . x x$
$658.4,659.2,659.3,674.2,674.3,668 . x x, 349 . x x$ 658.4, 659.2, 659.3, 674.2, 674.3, 668.xx, 349.xx
671.4, 673.0, 673.1, 673.2, 673.3, 518.xx (except $03,06,83,84,89), 998 . x x, 669.0,669.1,669.2$,

$669.3,669.4)$; Procedure codes not in (6831-6849, $6861-6869,689,99.0 x$ codes: '65420','65421','65423' codes: '65420',65421','65423'

MS-DRG: 767, 768, 774, 775 '7253','726','7271', '7279', '728','729'
Exclusion

Abnormal presentation, preterm, fetal death, multiple gestation diagnosis codes: '64420','64421',65100', '65101','65103','65110','65111','65113', '65120','65121', '65123,'65130',65131','65133','65140',65141', '65143', '65150,'65151',65153,'65160,'65161','65163,'65180', '65181','65183','65190','65191','65193','65220',65221', '65223', '65230',65231','65233','65240','65241','65243', '65260',65261', '65263',65640',65641',65643' '66050', '66051''66053''66230' '66231',66233',66960',66961', '67810','67811',67813',7615', 'V271','V272', V273', 'V274', '67810',67811,'67813',7615', 'V271,'V272', V273', 'V274',
'V275','V276','V277'

Breech procedure codes: '7251', '7252', '7253', '7254'

Abnormal presentation, preterm, fetal death, multiple gestation diagnoses, or breech procedure(ICD-9-CM codes as the above)

Previous Cesarean delivery diagnosis codes: '65420', '65421','65423'

MS-DRG: $765,766,767,768,774,775$ and diagnosis N/A

MS-DRG: 765, 766, 767, 768, 774, 775 and diagnosis

MS-DRG: 767, 768, 774, 775 and procedure codes: '720', '721', '7221', '7229', '7231','7239','724','7251',
Abnormal presentation, preterm, fetal death, multiple gestation diagnoses, or breech procedure(ICD-9-CM

Instrument-assisted delivery procedure codes: '720', '721', '7221', '7229', '7231', '7239','724','7251','7253','726', '7271','7279', '728','729'

N/A code as the above); 
SAS 9.3 (2011; Cary, NC: SAS Institute, Inc). The study was deemed exempt by the University of Hawai'i Institutional Review Committee. This research adhered to the Strengthening the Reporting of Observational Studies in Epidemiology (STROBE) guidelines for observational studies as outlined on http://www.strobe-statement.org.

\section{Results}

\section{Demographics}

In descriptive findings (Table 2), significant differences were seen across racial/ethnic groups for all demographic factors $(\mathrm{p}<.0001$ for all demographic comparisons). Native Hawaiians had the highest percentage of births for those under 20 years old (15.9\%) and Chinese had the lowest (1.4\%). Japanese has the highest percentage of births for those 40 years and older (8.3\%), while Native Hawaiians had the lowest (2.5\%).

Fifty-eight percent of the sample overall had private insurance, but this varied by racial/ethnic group, with 83.9\% of Japanese having private insurance (highest percent) compared to only $29.2 \%$ of other Pacific Islanders (lowest percent). Other Pacific Islanders (66.3\%) and Native Hawaiians $(50.8 \%)$ were most likely to have public insurance.

High-risk also varied across racial/ethnic groups with rates highest among Filipinos (39.2\%) and lowest in whites (35.5\%) and the other racial/ethnic group (33.7\%). Multiple gestations differed significantly across race/ethnicity with the highest rates seen in the Japanese (2.3\%) and the lowest rates seen in Native Hawaiians (1.2\%). Significant differences were seen in birth weight as well, with Filipinos having the highest percentage of low birth weight and other Pacific Islanders having the largest percentage of high birth weight. While percentages varied by racial/ethnic group, a total of $19.5 \%$ of patients were included multiple times, indicating they had more than one birth during the five-year study period.

\section{Clinical quality and safety outcomes}

Significant differences $(\mathrm{p}<.001)$ were seen for all birth outcomes by racial/ethnic subgroup (Table 3) except for obstetric trauma in Cesarean deliveries, which was extremely rare (less than 15 individuals total) and thus excluded from further analyses. Racial/ethnic differences were then compared in multivariable models (Table 4). Most of the significant differences seen by race/ethnicity in the descriptive analyses remained in multivariable models. Findings of interest are described below by Asian American and Pacific Islander subgroup.

\section{Chinese}

Chinese (5.7\%) had the highest percentage of obstetric trauma from vaginal deliveries without instruments. In multivariable models, Chinese women retained significantly higher rates of obstetric trauma for vaginal deliveries without instruments (Rate Ratio (RR): 1.95; 95\% CI: 1.53-2.48) compared to whites and Chinese also had significantly higher birth trauma in vaginal delivery with instrument (RR 1.42; 95\% CI:1.06, 1.91) in final models. In unadjusted analyses, Chinese also had the lowest unadjusted percentage (19.7\%) of Cesarean deliveries. However, this group did not vary significantly from whites for Cesarean deliveries in multivariable models.

\section{Japanese}

In unadjusted analyses, Japanese (4.5\%) had the second highest percentage of obstetric trauma from vaginal deliveries. In adjusted models, Japanese (RR: 1.52; 95\% CI: $1.27-1.81)$ had significantly higher rates of obstetric trauma for vaginal deliveries without instruments compared to whites and significantly lower (worse) rates of non-complicated births compared to whites (RR: 0.97; 95\% CI: 0.95-0.98). Japanese also had significantly lower rates of VBACs (RR:0.77; 95\% CI:0.63, 0.94) compared to whites.

\section{Native Hawaiians}

In unadjusted analyses, Native Hawaiians had the lowest percentage of obstetric trauma from both vaginal deliveries without instruments (1.6\%) and vaginal deliveries with instruments (12.6\%) across all racial/ethnic groups. In multivariable models, Native Hawaiians had significantly lower rates of obstetric trauma for vaginal deliveries without instruments (RR: 0.62; 95\% CI: 0.52-0.74) and with instruments (RR: 0.66; 95\%CI: 0.50-0.87) compared to whites. Native Hawaiians also had significantly higher (better) rates of non-complicated births compared to whites (RR: 1.01; 95\% CI: 1.00-1.02), the only studied group for which this was the case.

\section{Filipinos}

In unadjusted analyses, Filipinos had the highest percentage Cesarean deliveries (25.8\%). In multivariable models, Filipinos had significantly higher rates of Cesarean deliveries compared to whites (RR: 1.15; 95\% CI: 1.11-1.20) and significantly lower (worse) rates of non-complicated births compared to whites (RR: 0.96; 95\% CI: 0.95-0.97).

\section{Other Pacific Islanders}

In unadjusted analyses, other Pacific Islanders had the highest rates among all the racial/ethnic groups for all vaginal births after Cesarean (VBAC) $(25.7 \%)$ and uncomplicated VBACs (27.3\%). In multivariable models, other Pacific Islanders had significantly higher rates of all VBACs (RR: 1.28; 95\% CI: 1.08-1.51) and uncomplicated VBAC (RR: 1.23 ; 95\% CI: 1.03-1.46) compared to whites. Other Pacific Islanders also had significantly higher rates of Cesarean deliveries compared to whites 
Table 2 Demographics of childbirth hospitalizations in Hawai'i (2008-2012) from Hawai'i Health Information Corporation data

\begin{tabular}{|c|c|c|c|c|c|c|c|c|c|}
\hline & Chinese & Filipino & Native Hawaiian & Japanese & Other & Other Pacific Islander & White & Total & P-values ${ }^{*}$ \\
\hline Childbirth Hospitalizations & 2,335 & 14,965 & 17,081 & 7,888 & 10,126 & 8,326 & 15,004 & 75,725 & \\
\hline Patients & 1,968 & 12,545 & 13,094 & 6,608 & 8,904 & 6,366 & 12,831 & 62,316 & \\
\hline $\begin{array}{l}\text { Multiple maternal hospitalizations during study } \\
\text { period (\% from total \# of maternity-related } \\
\text { hospitalizations) }\end{array}$ & $686(29.4 \%)$ & $4,577(30.6 \%)$ & $7,145(41.8 \%)$ & $2,408(30.5 \%)$ & 2,969 (29.3\%) & $3,570(42.9 \%)$ & 4,224 (28.2\%) & $25,579(33.8 \%)$ & $<.0001$ \\
\hline \multirow{2}{*}{$\begin{array}{l}\text { \# patients with Multiple maternal hospitalizations } \\
\text { (\% from \# of patients) }\end{array}$} & $353(17.6 \%)$ & $2,252(17.8 \%)$ & $3,524(26.2 \%)$ & 1,227 (18.3\%) & $1,120(13.5 \%)$ & $1,665(25.9 \%)$ & $2,029(15.8 \%)$ & $12,170(19.5 \%)$ & $<.0001$ \\
\hline & N (\%) & N (\%) & N (\%) & N (\%) & N (\%) & N (\%) & N (\%) & N (\%) & P-values* \\
\hline Age & & & & & & & & & $<.0001$ \\
\hline$<20$ & $28(1.4 \%)$ & $1,029(8.2 \%)$ & $2,077(15.9 \%)$ & 207 (3.1\%) & 716 (8.0\%) & $695(10.9 \%)$ & 747 (5.8\%) & $5,499(8.8 \%)$ & $<.0001$ \\
\hline $20-29$ & $620(31.5 \%)$ & $5,971(47.6 \%)$ & 7,185 (54.9\%) & 1,887 (28.6\%) & 4,189 (47.0\%) & $3,672(57.7 \%)$ & $5,986(46.7 \%)$ & $29,510(47.4 \%)$ & $<.0001$ \\
\hline 30-39 & $1,176(59.8 \%)$ & 4,977 (39.7\%) & $3,510(26.8 \%)$ & $3,963(60.0 \%)$ & $3,667(41.2 \%)$ & $1,803(28.3 \%)$ & $5,452(42.5 \%)$ & 24,548 (39.4\%) & $<.0001$ \\
\hline $40+$ & $144(7.3 \%)$ & $568(4.5 \%)$ & $322(2.5 \%)$ & $551(8.3 \%)$ & $332(3.7 \%)$ & $196(3.1 \%)$ & $646(5.0 \%)$ & $2,759(4.4 \%)$ & $<.0001$ \\
\hline Payer & & & & & & & & & $<.0001$ \\
\hline Dept of Defense & $18(0.9 \%)$ & $216(1.7 \%)$ & $118(0.9 \%)$ & $72(1.1 \%)$ & 419 (4.7\%) & 75 (1.2\%) & $1,121(8.7 \%)$ & $2,039(3.3 \%)$ & $<.0001$ \\
\hline Private & $1,461(74.2 \%)$ & $8,550(68.2 \%)$ & $6,277(47.9 \%)$ & $5,542(83.9 \%)$ & 4,882 (54.8\%) & $1,861(29.2 \%)$ & 7,757 (60.5\%) & $36,330(58.3 \%)$ & $<.0001$ \\
\hline Public & $451(22.9 \%)$ & $3,711(29.6 \%)$ & $6,650(50.8 \%)$ & $776(11.7 \%)$ & $3,175(35.7 \%)$ & 4,221 (66.3\%) & 3,866 (30.1\%) & 22,850 (36.7\%) & $<.0001$ \\
\hline Self Pay & 38 (1.9\%) & $68(0.5 \%)$ & $49(0.4 \%)$ & $218(3.3 \%)$ & $428(4.8 \%)$ & 209 (3.3\%) & 87 (0.7\%) & $1,097(1.8 \%)$ & $<.0001$ \\
\hline \multicolumn{10}{|l|}{ Hospital } \\
\hline Urban & $1,831(93.0 \%)$ & $9,119(72.7 \%)$ & 7,737 (59.1\%) & $5,453(82.5 \%)$ & 6,322 (71.0\%) & $5,329(83.7 \%)$ & $7,252(56.5 \%)$ & $43,043(69.1 \%)$ & $<.0001$ \\
\hline \multicolumn{10}{|l|}{ High Risk } \\
\hline Yes & 706 (35.9\%) & $4,919(39.2 \%)$ & $4,907(37.5 \%)$ & 2,418 (36.6\%) & 3,005 (33.7\%) & $2,404(37.8 \%)$ & $4,558(35.5 \%)$ & 22,917 (36.8\%) & $<.0001$ \\
\hline \multicolumn{10}{|l|}{ Multiple Gestation } \\
\hline Yes & $33(1.7 \%)$ & $159(1.3 \%)$ & $159(1.2 \%)$ & $153(2.3 \%)$ & $115(1.3 \%)$ & $98(1.5 \%)$ & $263(2.1 \%)$ & $980(1.6 \%)$ & $<.0001$ \\
\hline Newborn birth weight & & & & & & & & & $<.0001$ \\
\hline $\operatorname{Low}(<2500 \mathrm{~g})$ & 155 (6.6\%) & 1,681 (11.2\%) & $1,306(7.6 \%)$ & 729 (9.2\%) & $752(7.4 \%)$ & 670 (8.0\%) & $917(6.1 \%)$ & $6,210(8.2 \%)$ & $<.0001$ \\
\hline Normal(2500-4000 g) & 2,093 (89.6\%) & $12,809(85.6 \%)$ & $14,486(84.8 \%)$ & 6,886 (87.3\%) & $8,777(86.7 \%)$ & 6,862 (82.4\%) & $12,676(84.5 \%)$ & 64,589 (85.3\%) & $<.0001$ \\
\hline $\operatorname{High}(>4000 \mathrm{~g})$ & $87(3.7 \%)$ & 475 (3.2\%) & 1,289 (7.5\%) & $273(3.5 \%)$ & $597(5.9 \%)$ & 794 (9.5\%) & $1,411(9.4 \%)$ & $4,926(6.5 \%)$ & $<.0001$ \\
\hline
\end{tabular}

on patient level (first visit). 
Table 3 Descriptive results from childbirth hospitalizations in Hawai'i (2008-2012) from Hawai'i Health Information Corporation data

\begin{tabular}{|c|c|c|c|c|c|c|c|c|c|}
\hline & $\begin{array}{c}\text { Chinese } \\
\mathrm{N}(\%)\end{array}$ & $\begin{array}{l}\text { Filipino } \\
\text { N (\%) }\end{array}$ & $\begin{array}{c}\text { Native Hawaiian } \\
\text { N (\%) }\end{array}$ & $\begin{array}{l}\text { Japanese } \\
\text { N (\%) }\end{array}$ & $\begin{array}{l}\text { Other } \\
\mathrm{N}(\%)\end{array}$ & $\begin{array}{l}\text { Other Pacific Islander } \\
\qquad \mathrm{N}(\%)\end{array}$ & $\begin{array}{l}\text { White } \\
\text { N (\%) }\end{array}$ & $\begin{array}{l}\text { Total } \\
\text { N (\%) }\end{array}$ & $\begin{array}{l}\text { P-values }{ }^{*} \\
\text { P-values }\end{array}$ \\
\hline \multicolumn{10}{|l|}{ Maternal quality indicator } \\
\hline Cesarean delivery rate & $414(19.7 \%)$ & 3,367 (25.8\%) & $3,509(22.9 \%)$ & $1,497(21.5 \%)$ & $2,297(25.2 \%)$ & $1,713(23.4 \%)$ & 3,293 (24.4\%) & $16,090(23.9 \%)$ & $<.0001$ \\
\hline Primary Cesarean delivery rate & $226(12.0 \%)$ & $1,713(15.4 \%)$ & $1,559(11.9 \%)$ & $820(13.2 \%)$ & $1,217(15.5 \%)$ & $877(14.3 \%)$ & $1,810(15.3 \%)$ & $8,222(14.1 \%)$ & $<.0001$ \\
\hline Vaginal Birth After Cesarean, all rate & $42(16.4 \%)$ & $316(14.2 \%)$ & $333(12.8 \%)$ & $114(12.9 \%)$ & $174(12.4 \%)$ & $357(25.7 \%)$ & $233(12.1 \%)$ & $1,569(14.7 \%)$ & $<.0001$ \\
\hline Vaginal Birth After Cesarean, Uncomplicated rate & $41(17.4 \%)$ & $310(15.0 \%)$ & $323(13.5 \%)$ & $108(13.1 \%)$ & $166(12.7 \%)$ & $349(27.3 \%)$ & $230(12.9 \%)$ & $1,527(15.4 \%)$ & $<.0001$ \\
\hline $\begin{array}{l}\text { Obstetric Trauma Rate - Vaginal Delivery Without } \\
\text { Instrument }\end{array}$ & $92(5.7 \%)$ & $276(2.8 \%)$ & $198(1.6 \%)$ & $242(4.5 \%)$ & $222(3.3 \%)$ & $151(2.7 \%)$ & $286(2.8 \%)$ & $1,467(2.8 \%)$ & $<.0001$ \\
\hline $\begin{array}{l}\text { Obstetric Trauma Rate - Vaginal Delivery With } \\
\text { Instrument }\end{array}$ & $44(29.7 \%)$ & $182(21.6 \%)$ & $69(12.6 \%)$ & $103(21.4 \%)$ & $110(21.2 \%)$ & $68(15.8 \%)$ & $123(18.8 \%)$ & $699(19.3 \%)$ & $<.0001$ \\
\hline Obstetric Trauma Rate - Cesarean delivery & $\begin{array}{l}\text { Too small } \\
\text { to report }\end{array}$ & $\begin{array}{l}\text { Too small } \\
\text { to report }\end{array}$ & $\begin{array}{l}\text { Too small } \\
\text { to report }\end{array}$ & $\begin{array}{l}\text { Too small } \\
\text { to report }\end{array}$ & $\begin{array}{l}\text { Too small } \\
\text { to report }\end{array}$ & $\begin{array}{l}\text { Too small } \\
\text { to report }\end{array}$ & $\begin{array}{l}\text { Too small } \\
\text { to report }\end{array}$ & $\begin{array}{l}\text { Too small } \\
\text { to report }\end{array}$ & 0.188 \\
\hline \multicolumn{10}{|l|}{ Non-complicated deliveries } \\
\hline ID Rate & $1,827(78.2 \%)$ & $11,671(78.0 \%)$ & $14,410(84.4 \%)$ & 6,166 (78.2\%) & $8,148(80.5 \%)$ & 6,498 (78.0\%) & $12,718(84.8 \%)$ & $61,438(81.1 \%)$ & $<.0001$ \\
\hline
\end{tabular}

${ }^{*}$-values were based on Chi-Square test or Fisher's exact tests for categorical variables; Kruskal-Wallis Test for continuous variables; $\mathrm{p}$-values were estimated assuming independence within patients. $\mathrm{N}$ (\%) for the above outcomes were based on hospitalizations. 
Table 4 Full models predicting rate ratios ((RR) [95\% CI]) for childbirth hospitalization outcomes in Hawai'i (2008-2012) from Hawai'i Health Information Corporation data

\begin{tabular}{|c|c|c|c|c|c|c|c|}
\hline & $\begin{array}{c}\text { Cesarean Delivery } \\
\text { Rate } \\
\text { RR }[95 \% \mathrm{Cl}]\end{array}$ & $\begin{array}{l}\text { Primary Cesarean } \\
\text { Delivery Rate }\end{array}$ & $\begin{array}{l}\text { Vaginal Birth } \\
\text { After Cesarean } \\
\text { Rate, All } \\
\text { RR }[95 \% \mathrm{Cl}]\end{array}$ & $\begin{array}{l}\text { Vaginal Birth After } \\
\text { Cesarean Rate, } \\
\text { Uncomplicated } \\
\text { RR }[95 \% \mathrm{CI}]\end{array}$ & $\begin{array}{c}\text { Obstetric Trauma } \\
\text { Rate - Vaginal Delivery } \\
\text { Without Instrument } \\
\text { RR }[95 \% \mathrm{Cl}]\end{array}$ & $\begin{array}{c}\text { Obstetric Trauma } \\
\text { Rate - Vaginal Delivery } \\
\text { With Instrument } \\
\text { RR }[95 \% \mathrm{Cl}]\end{array}$ & $\begin{array}{l}\text { Non-Complicated } \\
\text { Deliveries-ID rate }\end{array}$ \\
\hline \multicolumn{8}{|l|}{ Race/Ethnicity } \\
\hline Chinese vs. White & $0.96[0.88,1.05]$ & $1.00[0.88,1.14]$ & $0.79[0.58,1.08]$ & $0.78[0.57,1.06]$ & $1.95[1.53,2.48]$ & $1.42[1.06,1.91]$ & $0.97[0.95,0.99]$ \\
\hline Filipino vs. White & $1.15[1.11,1.20]$ & $1.11[1.04,1.18]$ & $0.97[0.82,1.13]$ & $0.91[0.78,1.07]$ & $1.04[0.88,1.23]$ & $1.07[0.87,1.31]$ & $0.96[0.95,0.97]$ \\
\hline Hawaiian vs. White & $1.02[0.98,1.06]$ & $0.83[0.78,0.88]$ & $0.99[0.84,1.17]$ & $0.94[0.79,1.11]$ & $0.62[0.52,0.74]$ & $0.66[0.50,0.87]$ & $1.01[1.00,1.02]$ \\
\hline Japanese vs. White & $0.96[0.91,1.02]$ & $1.00[0.92,1.08]$ & $0.84[0.73,1.03]$ & $0.77[0.63,0.94]$ & $1.52[1.27,1.81]$ & $1.02[0.80,1.29]$ & $0.97[0.95,0.98]$ \\
\hline Other vs. White & $1.14[1.09,1.19]$ & $1.16[1.09,1.25]$ & $0.87[0.73,1.05]$ & $0.82[0.68,0.99]$ & $1.21[1.01,1.44]$ & $1.06[0.84,1.33]$ & $0.98[0.97,0.99]$ \\
\hline Other PI vs. White & $1.16[1.10,1.22]$ & $1.16[1.07,1.26]$ & $1.28[1.08,1.51]$ & $1.23[1.03,1.46]$ & $1.09[0.89,1.34]$ & $0.80[0.60,1.06]$ & $0.97[0.96,0.98]$ \\
\hline \multicolumn{8}{|l|}{ Age Groups } \\
\hline$<20$ vs. $40+$ & $0.47[0.44,0.51]$ & $0.80[0.72,0.88]$ & $1.96[1.39,2.78]$ & $2.02[1.41,2.89]$ & $1.76[1.26,2.46]$ & $1.30[0.85,1.99]$ & $0.90[0.88,0.92]$ \\
\hline $20-29$ vs. $40+$ & $0.60[0.57,0.63]$ & $0.66[0.61,0.72]$ & $1.55[1.21,1.98]$ & $1.50[1.15,1.95]$ & $1.32[0.98,1.78]$ & $1.41[0.99,2.01]$ & $0.96[0.94,0.97]$ \\
\hline $30-39$ vs. $40+$ & $0.75[0.72,0.79]$ & $0.71[0.66,0.77]$ & $1.31[1.03,1.67]$ & $1.32[1.02,1.71]$ & $1.15[0.85,1.55]$ & $1.23[0.87,1.74]$ & $0.99[0.98,1.01]$ \\
\hline \multicolumn{8}{|l|}{ Payer } \\
\hline DOD vs. private & $0.98[0.90,1.07]$ & $0.87[0.77,0.99]$ & $0.88[0.67,1.14]$ & $0.88[0.68,1.14]$ & $0.74[0.55,1.00]$ & $0.64[0.41,0.98]$ & $1.06[1.04,1.08]$ \\
\hline Public vs. private & $0.98[0.95,1.01]$ & $0.84[0.81,0.88]$ & $1.23[1.10,1.37]$ & $1.25[1.11,1.41]$ & $0.54[0.48,0.61]$ & $0.76[0.65,0.90]$ & $1.02[1.01,1.03]$ \\
\hline Self Pay vs. private & $0.84[0.76,0.94]$ & $0.82[0.70,0.97]$ & $1.28[0.87,1.88]$ & $1.23[0.82,1.86]$ & $0.90[0.63,1.28]$ & $1.09[0.73,1.62]$ & $0.98[0.95,1.01]$ \\
\hline \multicolumn{8}{|l|}{ High Risk } \\
\hline Yes vs. No & $1.52[1.48,1.55]$ & $2.43[2.25,2.43]$ & $0.70[0.63,0.78]$ & $0.74[0.67,0.82]$ & $1.14[1.03,1.28]$ & $1.04[0.90,1.20]$ & $0.98[0.97,0.99]$ \\
\hline \multicolumn{8}{|l|}{ Hospital } \\
\hline rural vs. urban & $1.66[1.61,1.71]$ & $1.73[1.66,1.81]$ & $0.09[0.07,0.12]$ & $0.10[0.07,0.13]$ & $0.81[0.71,0.92]$ & $0.65[0.54,0.77]$ & $1.14[1.13,1.14]$ \\
\hline \multicolumn{8}{|l|}{ Multiple Gestation } \\
\hline Yes vs. No & $N A^{1}$ & NA & $0.34[0.15,0.80]$ & NA & $0.94[0.47,1.87]$ & $1.46[0.82,2.59]$ & $0.98[0.97,1.03]$ \\
\hline \multicolumn{8}{|l|}{ Baby Birth Weight } \\
\hline Low vs. Normal & $1.20[1.10,1.31]$ & $1.11[0.97,1.26]$ & $1.22[0.90,1.65]$ & $1.17[0.87,1.59]$ & $0.53[0.42,0.67]$ & $0.75[0.57,0.99]$ & $0.98[0.96,1.01]$ \\
\hline High vs. Normal & $1.06[0.97,1.16]$ & $0.83[0.73,0.95]$ & $1.25[0.92,1.69]$ & $1.21[0.89,1.63]$ & $0.32[0.25,0.41]$ & $0.47[0.33,0.65]$ & $1.04[1.02,1.06]$ \\
\hline
\end{tabular}

${ }^{1} \mathrm{~N} / \mathrm{A}$ : For cesarean delivery Rate, primary cesarean delivery rate and vaginal birth after cesarean rate, uncomplicated, multiple gestations were excluded out of analyses based on AHRQ quality indicators. 
(RR: 1.16; 95\% CI: 1.10-1.22). In final models, other Pacific Islanders had significantly lower (worse) rates of non-complicated births compared to whites (RR: 0.97; 95\% CI: 0.96-0.98).

\section{Discussion}

Our study hypothesis was that we would see poorer maternal quality and safety outcomes for Native Hawaiians, Filipinos, and other Pacific Islanders compared to whites. Indeed, high rates of Cesarean deliveries were seen among Filipinos and other Pacific Islanders. Also, Filipinos and other Pacific Islanders had significantly higher percentages of complicated births overall compared to whites. These findings reveal poorer outcomes across some maternal quality and safety measures for Filipinos and other Pacific Islanders compared to whites.

However, many maternal quality and safety outcomes were better for Native Hawaiians compared to whites. Native Hawaiians had significantly lower rates of obstetric trauma during vaginal delivery compared to whites and were the only racial/ethnic group studied to have significantly lower percentages of complicated births overall compared to whites. Nor were all maternal quality and safety outcomes worse for other Pacific Islanders. Instead, other Pacific Islanders had VBACs, a desirable outcome [19], at significantly higher rates.

These findings are intriguing. One possible reason could be that the references groups of whites in Hawai'i may have poorer health outcomes compared to Whites on the continental US. Certainly, the Caucasian population in Hawai' $i$ is uniquely distributed, including many who have come from the continental US and only remain temporarily for military service, retirement, and employment [23]. Additionally, whites are not typically the healthiest group in Hawai' $i$ as the health status and life expectancy of the Caucasian population tends to fall below that of the Chinese and Japanese, though typically above that of Native Hawaiians [28]. That said, our findings are supported by previous work in Hawai'i and elsewhere has found that, despite having higher risk factors for poor outcomes, Native Hawaiians and other Pacific Islander have low rates of some adverse perinatal outcomes (including low birth weight) $[11,29,30]$. This suggests that Native Hawaiians both in Hawaii $i$ and other locations may have strong protective factors that support positive birth outcomes, such as strong community and family ties [31]. These findings could be related to a literature in the US that has demonstrated low rates of adverse birth outcomes among some disadvantaged (primarily Hispanic) immigrant groups [32].

We also found poor outcomes for some maternal quality and safety measures among Chinese and Japanese women compared to whites. Japanese women had significantly higher percentages of complicated births overall and high rates of obstetric trauma were seen among Chinese and Japanese women with vaginal deliveries. These are interesting findings as Japanese and Chinese populations in Hawai'i tend to have favorable health profiles, good access to care, and high insurance coverage rates $[23,28]$. However, the older populations who show strong longevity may have distinct demographic characteristics compared to those who are young enough to be having children [23], or other risk factors such as English proficiency may be at play $[33,34]$. These may also reflect cultural preferences. These are important areas for further study as they suggest distinct intervention and policy solutions.

Our findings support previous research that found high rates of perineal trauma in Asians [12,14,35] and add new evidence about this issue. For instance, we find that unadjusted rate of obstetric trauma with an instrument was high among Filipinos in unadjusted analyses (higher than in all other racial/ethnic groups except Chinese). However, the unadjusted rate of obstetric trauma without an instrument was not significantly higher among Filipinos compared to other studied racial/ethnic groups in descriptive analyses.

Little research exists on perinatal outcomes for Pacific Islanders compared to Asian American subgroups in the published literature [12-14]. Previous research has aggregated Native Hawaiian with other Pacific Islander groups [12]. We find notable differences between Native Hawaiians and other Pacific Islanders, particularly in Cesarean delivery rates, which are significantly higher for other Pacific Islanders compared to whites in multivariable models, a difference not seen for Native Hawaiians. As the average weight and height of Pacific Islander women are higher than for many other Asian women [36], this is not surprising. However, this cannot account for the findings of lower primary Cesarean delivery rates in Native Hawaiians. This may be an important area for further study.

Cesarean sections are the most common major surgical procedure in the United States [37]. Promoting appropriate use of Cesarean delivery is a particular focal area for quality improvement [6,38]. Our research suggests that a better understanding of this topic in Filipinos and other Pacific Islanders may be fruitful toward a goal of Cesarean delivery reduction. The increase in Cesarean section deliveries have been coupled with a decrease in the rate of VBACs [39]. In our study, we see rates of VBACs are very high among other Pacific Islander women.

Our findings of significant variation across Asian and Pacific Islander subgroups for study outcomes have important implications for policy and practice. Because much research combines Asian American and Pacific Islander women together, it can be challenging to understand the specific health risks for subgroup for 
research and clinical practice [12-14]. Thus, not only does our study highlight the importance of variation, but highlights particular areas for improvement for each racial/ethnic group to potentially improve on these outcomes. This becomes increasing more important as the number of Asian American and Pacific Islander women grow in the U.S.

For example, a recent overview of these metrics using aggregated Asian American and Pacific Islander groups found that "API [Asian and Pacific Islander] women had higher rates of obstetric trauma, both with and without instrument assistance, than other women [15]". Our study finds considerable differences in this metric for Asian and Pacific Islander subgroups. Chinese (5.7\%) and Japanese $(4.5 \%)$ had the highest percentage of obstetric trauma from vaginal deliveries without instruments while Hawaiians had the lowest percentages $(1.6 \%)$ in all groups. Filipinos were only high in obstetric trauma with an instrument, but not in obstetric trauma without an instrument compared to other studied racial/ ethnic groups. This information suggests different foci for quality improvement efforts for distinct Asian and Pacific Islander groups.

We found higher rates of complicated deliveries in several Asian and Pacific Islander groups compared to whites. Complicated deliveries are considerably more expensive than uncomplicated deliveries [40]. Our findings may be of particular interest to Medicaid, which cared for $37 \%$ of the total births and considerably higher percentages of some groups (e.g., other Pacific Islanders) who had poor outcomes. At the same time, obstetric trauma rates were particularly high among Japanese individuals who are primarily insured by private providers in our study. Obstetric trauma can lead to clinical and quality of life issues over time [41] so reducing this risk may be a focus of quality improvement in private providers with large numbers of Japanese members.

\section{Limitations}

This study has several important strengths. We have a full sample with all civilian hospitalizations in a diverse state with detailed race/ethnicity data for five years. Hawai' $i$ hospitalization data is extremely diverse, including racial/ethnic groups not easily captured in most population-based samples, but that have increasing relevance to the U.S. population generally due to their increasing size [9]. A major strength of the Hawai'i data is that, because of the state's unique demographic composition, it provides a sufficient sample size to offer a window into the utilization patterns of understudied groups in a timely way while these populations are increasing in other locations. Findings from Hawai'i can be compared to other settings in the continental US and elsewhere and may provide benchmarks for locations where Asian and Pacific Islander subgroups analyses would not currently be reliable without extremely large samples. Thus, information about maternal health outcomes in Hawai' $i$ is of relevance to that state, but also provides key comparative data for other locations. Our findings may be particularly informative for states, like California and New York, and for specific communities, such as New York City, San Diego, and Houston, where Asian and/or Pacific Islander populations are particularly high and growing [10].

However, this study also has some limitations. Using administrative data generally and the AHRQ metrics specifically have many advantages as these provide a comprehensive, low burden, low cost method to obtain standardized information on safety events across large populations that incorporate risk and have been developed with extensively clinical and research expertise [21]. These metrics are also readily comparable across multiple locations in the US and even internationally [19-21]. However, administrative data has some potential disadvantages. Our data lacks some relevant demographic information, such as education or health literacy, as well as some relevant clinical data, such as women's previous birth histories, gestation week, and BMI. These factors could certainly help to explain some of the findings across racial/ethnic groups and may suggest critical areas for interventions [42]. Also, though our models account for public vs. private insurance and maternal age, we are not able to fully adjust for sociodemographic differences between groups. This could be relevant in that Chinese and Japanese women tend to have higher socioeconomic status compared to Native Hawaiian and Pacific Islander women, which may account for some of our findings [23].

At the same time, understanding how patterns of disparities manifest by race/ethnicity is important, even if these patterns can ultimately be explained by other socio-demographic and clinical factors. Additionally, our data provides baseline information about racial/ethnic patterns in a location with large Asian and Pacific Islander populations that could be potentially compared not only to locations with large numbers of these groups, but also in locations that have smaller numbers of these population groups, but who already compile the AHRQ metrics in their state $[39,43]$.

Also, we only had data on maternal ethnicity and were thus unable to examine the effect that paternal ethnicity may have on perinatal outcomes. We also lack information about maternal birthplace, which may be an issue as foreign-born women may have better outcomes than those born in the US [44]. Additionally, the race/ethnicity variables have limitations. We only report only primary race/ethnicity and our findings may not be generalizable to those of multiple Asian ethnicities [14]. Also, problems in 
concordance have been found between self-reported race/ ethnicity and race/ethnicity recorded in administrative data sets, particularly for minority races $[45,46]$. While the HHIC data has strong and consistent protocols in data collection across hospitals in Hawai i, it is possible that the racial/ethnic classifications used in this study may not precisely mirror the classifications individuals would choose for themselves. However, the research on this topic concludes that these concordance issues likely serve to underestimate disparities [45]. Thus, better measurement of race/ethnicity would likely only find more of disparities demonstrated here rather than less.

We followed AHRQ guidelines in our metrics, making our research comparable to the extensive existing literature on this topic. However, the AHRQ Cesarean measures have been criticized for excluding many patients with risk factors from the measure instead of risk adjusting for them, which may impact study analyses by race/ ethnicity [47]. Also, our outcomes are only indicators of quality, not definitive definitions [48]. Self-reported experiences of health care quality might vary.

\section{Conclusions}

Our study provides a comprehensive portrait of variation in quality and safety outcomes by race/ethnicity across diverse Asian and Pacific Islander racial/ethnic groups. We identify some potential disparities in maternal health outcomes, particularly in obstetric trauma for Chinese and Japanese and Cesarean rates for Filipinos and other Pacific Islanders. This knowledge will help improve the specificity of quality data around maternal outcomes and identify important groups to target for interventions.

Our study also highlights that Asian and Pacific Islanders subgroups must be disaggregated to understand patterns, identify possible disparities, and design effective interventions. This is particularly important to consider in childbirth, which is the most common reason for hospital admission among women. While disparities for Asian or Pacific Islander groups are not uniform, understanding distinct risks and problematic birth outcomes among groups is critical to ensuring health equity among all racial/ethnic groups.

\section{Abbreviations}

AHRQ: Agency for healthcare research and quality; ANOVA: Analysis of variance; API: Asian and pacific islander; Cl: Confidence intervals; DOD: Department of defense; GEE: Generalized estimating equations; HHIC: Hawai'i health information corporation; ICD-9: International classification of diseases $-9^{\text {th }}$ revision - clinical modification codes; ID: "Ideal delivery"; IOM: Institute of medicine; MSDRGs: Medicare severity-diagnostic related groups; NA: Not applicable; RR: Rate ratio; STROBE: Strengthening the reporting of observational studies in epidemiology; US: United states; VBAC: Vaginal births after cesarean.
}

\section{Competing interests}

The authors declare that they have no competing interests.

\section{Authors' contribution}

TS conceived and designed the study, obtained the funding, and drafted the manuscript. AC participated in the study design and coordination and helped to draft the manuscript. YC participated in the design of the study and performed the statistical analysis. JM obtained the data, participated in the study design and coordination, and helped to draft the manuscript. All authors read and approved the final manuscript.

\section{Acknowledgements}

This project was supported by Agency for Healthcare Research and Quality (AHRQ) grant R21 HS021903. The project was also partially supported by $\mathrm{NIH}$ Awards: U54MD007584, G12MD007601, 8P20GM103466-11, and AHRQ grant RO1HS019990.

\section{Author details}

1'Office of Public Health Studies, University of Hawai'i at Mānoa, 1960 East-West Road, Biomed T102, Honolulu, HI 96822, USA. ²Department of OB/ GYN, John A. Burns School of Medicine, University of Hawai'i, 1319 Punahou St, Suite 824, Honolulu, HI 96826, USA. ${ }^{3}$ Biostatistics Core, John A. Burns School of Medicine, University of Hawai'i, 651 Ilalo Street, Biosciences Building, Suite 211, Honolulu, HI 96813, USA. ${ }^{4}$ Hawai'i Health Information Corporation, 733 Bishop St, Honolulu, HI 96813, USA.

Received: 16 May 2014 Accepted: 27 August 2014

Published: 30 August 2014

\section{References}

1. Podulka J, Stranges E, Steiner C: Hospitalizations related to childbirth, 2008. April 2011 HCUP statistical brief \#110. [http://www.hcup-us.ahrq.gov/ reports/statbriefs/sb110.jsp]

2. Bezruchka S: The deteriorating international ranking of U.S. health status. Annu Rev Public Health 2012, 33:157-173.

3. Kim D, Saada A: The social determinants of infant mortality and birth outcomes in Western developed nations: a cross-country systematic review. Int J Environ Res Public Health 2013, 10:2296-335.

4. Tucker MJ, Berg CJ, Callaghan WM, Hsia J: The Black-White disparity in pregnancy-related mortality from 5 conditions: differences in prevalence and case-fatality rates. Am J Public Health 2007, 97:247-51.

5. Anachebe NF: Racial and ethnic disparities in infant and maternal mortality. Ethn Dis 2006, 16(2 Suppl 3):S3-71-6. http://www.ncbi.nlm.nih. gov/pubmed/16774028.

6. Adams K, Corrigan JM (Eds): Priority Areas for National Action: Transforming Health Care Quality. Washington, DC: the National Academies Press; 2003. http://www.nap.edu/catalog.php?record_id=10593.

7. Institute of Medicine (US) Committee on Quality of Health Care in America: Crossing the Quality Chasm: A New Health System for the 21st Century. Washington (DC): National Academies Press (US); 2001.

8. Agency for Healthcare Research and Quality: Patient Safety and Quality: An Evidence-based Handbook for Nurses. Rockville, MD: Agency for Healthcare Research and Quality; 2008. http://www.ahrq.gov/professionals/cliniciansproviders/resources/nursing/resources/nurseshdbk/index.html.

9. US Census Bureau: The Native Hawaiian and Other Pacific Islander population 2010. 2012, http://www.census.gov/prod/cen2010/briefs/ c2010br-12.pdf.

10. US Census Bureau: 2013 Facts for Features. Asian/Pacific American Heritage Month: May 2013. http://www.census.gov/newsroom/releases/ pdf/cb13ff-09_asian.pdf.

11. Centers for Disease Control and Prevention (CDC): Maternal, pregnancy, and birth characteristics of Asians and Native Hawaiians/Pacific Islanders-King County, Washington, 2003-2008. MMWR Morb Mortal Wkly Rep 2011, 60:211-213.

12. Rao AK, Daniels K, El-Sayed YY, Moshesh MK, Caughey AB: Perinatal outcomes among Asian American and Pacific Islander women. Am J Obstet Gynecol 2006, 195:834-8.

13. Rao AK, Cheng YW, Caughey AB: Perinatal complications among different Asian-American subgroups. Am J Obstet Gynecol 2006, 194:e39-41.

14. Wong LF, Caughey AB, Nakagawa S, Kaimal AJ, Tran SH, Cheng YW: Perinatal outcomes among different Asian-American subgroups. Am J Obstet Gynecol 2008, 199(4):382.e1-6. doi:10.1016/j.ajog.2008.06.073. Epub 2008 Aug 23. 
15. Agency for Healthcare Research and Quality: Disparities in Health Care Quality Among Minority Women: Findings From the 2011 National Healthcare Quality and Disparities Reports. Rockville, MD: AHRQ Publication No. 12-00063-EF, October 2012. Agency for Healthcare Research and Quality; http:// www.ahrq.gov/qual/nhqrdr11/nhqrminoritywomen11.htm.

16. Nguyen BT, Cheng YW, Snowden JM, Esakoff TF, Frias AE, Caughey AB: The effect of race/ethnicity on adverse perinatal outcomes among patients with gestational diabetes mellitus. Am J Obstet Gynecol 2012, 207:322.e1-6. doi:10.1016/j.ajog.2012.06.049. Epub 2012 Jun 29.

17. Cabacungan ET, Ngui EM, McGinley EL: Racial/ethnic disparities in maternal morbidities: a statewide study of labor and delivery hospitalizations in Wisconsin. Matern Child Health J 2012, 16:1455-67.

18. Handa VL, Danielsen BH, Gilbert WM: Obstetric anal sphincter lacerations. Obstet Gynecol 2001, 98:225-230.

19. AHRQ: AHRQ Quality Indicators ${ }^{\mathrm{TM}}$ Toolkit for Hospitals: Fact Sheet. Publication \# 12-M038-E. http://www.ahrq.gov/research/findings/ factsheets/quality/qifactsheet/index.html.

20. Raleigh VS, Cooper J, Bremner SA, Scobie S: Patient safety indicators for England from hospital administrative data: case-control analysis and comparison with US data. BMJ 2008, 17:a1702.

21. Center for Health Policy/Center for Primary Care and Outcomes Research \& Battelle Memorial Institute: Quality Indicator Measure Development, Implementation, Maintenance, and Retirement (Prepared by Battelle, under Contract No. 290-04-0020). Rockville, MD: Agency for Healthcare Research and Quality; 2011. http://qualityindicators.ahrq.gov/Downloads/Resources/ Publications/2011/QI_Measure_Development_Implementation_ Maintenance_Retirement_Full_5-3-11.pdf.

22. Ghosh C: Healthy People 2010 and Asian Americans/Pacific Islanders: defining a baseline of information. Am J Public Health 2003, 93:2093-2098.

23. Andrade N, McDermott J (Eds): People and cultures of Hawai'i: The evolution of culture and ethnicity. Honolulu, HI: The University of Hawai'i Press; 2011.

24. Tsai PJ, Roberson E, Dye T: Gestational diabetes and macrosomia by race/ ethnicity in Hawaii. BMC Res Notes 2013, 6:395.

25. Mau MK, Sinclair K, Saito EP, Baumhofer KN, Kaholokula JK: Cardiometabolic health disparities in Native Hawaiians and other Pacific Islanders. Epidemiol Rev 2009, 31:113-129.

26. Hawaili Health Information Corporation Inpatient Data. http://www.hhic.org/.

27. Gregory KD, Fridman M, Shah S, Korst LM: Global measures of quality- and patient safety-related childbirth outcomes: should we monitor adverse or ideal rates? Am J Obstet Gynecol 2009, 200(6):681.e1-7. doi:10.1016/j. ajog.2009.02.033.

28. Park CB, Braun KL, Horiuchi BY, Tottori C, Onaka AT: Longevity disparities in multiethnic Hawaii: an analysis of 2000 life tables. Public Health Reports 2009, 124:579-84.

29. Kieffer EC, Mor JM, Alexander GR: The perinatal and infant health status of Native Hawaiians. Am J Public Health 1994, 84:1501-4.

30. Le LT, Kiely $\mathrm{L}$, Schoendorf KC: Birthweight outcomes among Asian American and Pacific Islander subgroups in the United States. Int J Epidemiol 1996, 25:973-9.

31. Look MA (Ed): Assessment and Priorities for Health \& Well-Being in Native Hawaiians \& other Pacific Peoples: Publisher: Center for Native \& Pacific Health Disparities Research, University of Hawai'i; http://blog.hawaii.edu/ uhmednow/files/2013/09/AP-Hlth-REPORT-2013.pdf.

32. Hoggatt KJ, Flores M, Solorio R, Wilhelm M, Ritz B: The "Latina epidemiologic paradox" revisited: the role of birthplace and acculturation in predicting infant low birth weight for Latinas in Los Angeles, CA. J Immigr Minor Health 2012, 14:875-884.

33. Parsons L, Day S: Improving obstretric outcomes in ethnic minorities: An evaluation of health advocacy in Hackney. J Publ Health Med 1992, 14:183-91.

34. Ro M: Moving forward: Addressing the health of Asian American and Pacific Islander. Women. Am J Public Health 2002, 92:516-519.

35. Wheeler J, Davis D, Fry M, Brodie P, Homer CS: Is Asian ethnicity an independent risk factor for severe perineal trauma in childbirth? A systematic review of the literature. Women Birth 2011, 25:107-113.

36. Davis J, Busch J, Hammatt Z, Novotny R, Harrigan R, Grandinetti A, Easa D: The relationship between ethnicity and obesity in Asian and Pacific Islander populations: a literature review. Ethn Dis 2004, 14:111-8.

37. Hoyert DL, Freedman MA, Strobino DM, Guyer B: Annual summary of vital statistics: 2000. Pediatrics 2001, 108:1241-55.
38. Bailit JL, Garrett JM, Miller WC, MCMahon MJ, Cefalo RC: Hospital primary cesarean delivery rates and the risk of poor neonatal outcomes. Am J Obstet Gynecol 2002, 187:721-7.

39. Russo CA, Wier L, Steiner C: Hospitalizations related to childbirth, 2006, HCUP Statistical Brief, 71. Rockville, MD: Agency for Healthcare Research and Quality; 2009. http://www.hcup-us.ahrq.gov/reports/statbriefs/sb71.jsp.

40. Agency for Health Care Research and Quality: Most American Women Experience Complications During Delivery: AHRQ News and Numbers; 2011. http://www.ahrq.gov/news/nn/nn051911.htm.

41. Haadem K, Ohrlander S, Lingman G: Long-term ailments due to anal sphincter rupture caused by delivery-a hidden problem. Eur J Obstet Gynecol Reprod Biol 1988, 27:27-32.

42. Aune D, Saugstad OD, Henriksen T, Tonstad S: Maternal body mass index and the risk of fetal death, stillbirth, and infant death: a systematic review and meta-analysis. JAMA 2014, 311:1536-46.

43. Louisiana Department of Health and Hospitals: Louisiana Cesarean Rates. 2012. http://www.cesareanrates.com/storage/state_pdfs/Louisiana_Cesarean_ Rates_Report.pdf.

44. Singh GK, Yu SM: Adverse pregnancy outcomes: differences between US- and foreign-born women in major US racial and ethnic groups. Am J Public Health 1996, 86:837-43.

45. Kressin NR, Chang BH, Hendricks A, Kazis LE: Agreement between administrative data and patients' self-reports of race/ethnicity. Am J Public Health 2003, 93:1734-1739.

46. Boehmer U, Kressin NR, Berlowitz DR, Christiansen CL, Kazis LE, Jones JA: Self-reported vs administrative race/ethnicity data and study results. Am J Public Health 2002, 92:1471-1472.

47. Huesch MD, Currid-Halkett E, Doctor JN: Measurement and risk adjustment of prelabor cesarean rates in a large sample of California hospitals. Am J Obstet Gynecol 2014, 210(5):443.e1-443.e17. doi:10.1016/j.ajog.2013.12.007. Epub 2013 Dec 4

48. OSHPD: OSHPD implementation of AHRQ Inpatient Mortality Indicators, 20062007. 2011. http://www.oshpd.ca.gov/HID/Products/PatDischargeData/ AHRQ/iqi/TechNOTE.pdf.

\section{doi:10.1186/1471-2393-14-298}

Cite this article as: Sentell et al:: Maternal quality and safety outcomes for Asians and Pacific Islanders in Hawai'i: an observational study from five years of statewide data. BMC Pregnancy and Childbirth 2014 14:298.

\section{Submit your next manuscript to BioMed Central and take full advantage of:}

- Convenient online submission

- Thorough peer review

- No space constraints or color figure charges

- Immediate publication on acceptance

- Inclusion in PubMed, CAS, Scopus and Google Scholar

- Research which is freely available for redistribution 